\title{
A Morphology based Approach for Segmentation and Tumor Detection from MRI Images in Noisy Environment and Performance Evaluation
}

\author{
Gopi Karnam ${ }^{1}$, Ramashri Thirumala ${ }^{2}$ \\ Assistant Professor, Department of ECE, Sri Venkateswara University College (SVU) of Engineering, Tirupati, \\ Andhra Pradesh, India ${ }^{1}$
}

Professor, Dept of ECE, Sri Venkateswara University College (SVU) of Engineering, Tirupati, Andhra Pradesh, India ${ }^{2}$

\begin{abstract}
In the field of medical image handling, location of brain tumor from Magnetic resonance image (MRI) examine has become to be an active amongst the most dynamic exploration. Detection of the tumor is the fundamental goal of the framework. In this paper, MRI brain image is utilized to tumor recognition process. This framework incorporates test the brain image process, image separating, segmentation, and morphological operation, tumor detection. In recent years many image processing procedures are widely used on medical images to detect tumors at an early and treatment stages. When the images are added with noise the difficulties are primarily associated with the detection of tumors. In such cases the intensity thresholding might fail to identify the tumors. Edges characterize boundaries are the fundamental importance in image processing. The image edge detection reduces the data by filtering and preserving the important structural attributes. So understanding the edge detection algorithms is essential. Here the morphology based region of interest segmentation combined with watershed transform of MRI tumor image is performed and comparative analysis in noisy environment such as Gaussian, salt and pepper, Poisson and speckle is performed. Here different edge detection filters such as average, Gaussian and laplacian, sobel and prewit, unsharp and $\log$ in presence of noise. The parameters such as Cross correlation, Peak signal to noise ratio, Mean absolute error for different operators are evaluated. The motivation behind the morphological operators is to separate the tumor part of the image. In this paper, the brain image is considered for examination and recognition. At first the region of interest is found, that recognizes the specific substance of the image and set the boundary of it. Morphological operations are utilized for edge identification. The four parameters, such as Probabilistic Rand Index (PRI), Variation of Information (VOI), Global Consistency Error (GCE), Structural similarity (SSIM) have been utilized to evaluate the performance of segmentation.
\end{abstract}

Keywords: Magnetic Resonance Image (MRI), Segmentation, Noise, Morphological Operation, Watershed Segmentation, Brain Tumor.

\section{INTRODUCTION}

A tumor can be characterized as a mass which develops with no control of ordinary forces. Diagnosis of tumors by using more reliable algorithms been the main focus of the latest developments in the medical imaging of brain tumor in MRI images has been the active research. The partition of the cells and their centers from whatever leftovers of the image substance is one of the rule issues confronted by the most part of the medical image diagnosis systems. The procedure of partition i.e. segmentation, is paid at most significance in the development of a powerful and robust diagnosis system. Image segmentation is the major issue in tumor detection. Various techniques have been proposed in the past for brain tumor detection. Exact measurements in brain diagnosis are quite difficult on account of various shapes, sizes and appearances of tumors. Tumors can develop causing defects in neighbouring tissues also, which gives an overall irregular structure for healthy tissues as well. In this paper we have built up a algorithm which can recognize the tumor inside of the brain in images which are acquired through magnetic imaging resonance (MRI). The images which are gotten through this methodology are in a standard arrangement that is digital imaging and communication for medicine (DICOM). This is a standard configuration for all the medical pictures. It was developed by the national electronic manufactures association. This standard formats mainly used for storing, printing and transmitting information in medical imaging. These images are taken and changed over into greyscale images for pre-handling which incorporate a percentage of the imaging enhancement strategies and later on post processing which incorporate morphological operations. Segmentation is an imperative step in the processing of MR images with the goal of restorative analysis, 3-D representation of the human brain[1]. Morphological operations here have focal influence in the location of the tumor. In image handling morphological operations are utilized for analyzing and segmentation of various examples in a image. Therefore 
Vol. 5, Issue 12, December 2016

by using this algorithm we are able to segment the tumors and also we are able to clearly observe the shape of the tumors. Image segmentation is the process of partitioning an advanced image into various segments (sets of pixels, otherwise called super pixels).

The objective of segmentation is to streamline and/or change the representation of a image into something that is more important and simpler to analyze. Image segmentation is ordinarily used to find objects and boundaries (lines, curves etc.)in pictures. All the more precisely, Image segmentation is the procedure of assigning a label to each pixel in a Image such that pixels with the same name share certain visual attributes.

In case of medical image segmentation the aim is to:

$>$ Study anatomical structure

$>$ Identify Region of Interest i.e. find tumor, lesion and other variations from the norm

$>$ Measure tissue volume to measure growth of tumor and help in treatment planning before radiation therapy.

In this paper morphology based region of interest segmentation combined with watershed transform on DICOM image is performed and comparative analysis in noisy environment such as Gaussian, Salt and pepper, Poisson and speckle is performed. Here different edge detection filters such as average, Gaussian, laplacian and sobel, prewitt, Log in presence of noise is used. The results are helpful to study and analyse the influence of noise on DICOM images while extracting region of interest and to know how effectively the operators are able to detect, overcoming the impact of different noise. In real world applications gained medical images contain object boundaries and object shadow and noise. The processing to be connected relies on the content which one desires to extract. Therefore a frequent issue in low level vision arises to eliminate and suppress the noise and other details from the degraded image, without degrading the region of interest. The medical image identification is an important work for object recognition of the human organs such as lungs, brain tumors, ribs and it is an essential preprocessing step in medical image segmentation. The segmentation of brain tumor from magnetic resonance images is an important but time-consuming task performed by medical experts [4]. Backbone and the driving force behind the imaging workflow of image display in modern era is the DICOM (Digital Imaging and Communications in Medicine) standard.

MRI is fundamentally utilized as a part of the biomedical to distinguish and visualize better details in the inner structure of the body. This technique is fundamentally utilised to identify the distinctions in the tissues which have an obviously better method when contrasted with computed tomography (CT). So this makes this system an exceptionally unique one for the mind tumor recognition and disease imaging. [2]. Edge recognition will prompt finding the accurate location of tumor.[5]In medical imaging, division of tissues and structure acting a very important role in many image analysis applications urbanized for medical diagnosis [1].

An accurate, reliable, and automatic segmentation of these tissues and structures can improve diagnosis and treatment of brain diseases [4, 5]. Precise size in brain analysis is troublesome due to various shapes and sizes of tumor [2, $3]$. The best type of imaging to analyze most types of brain tumors is MRI [8,9] this system is essentially used to recognize the distinctions in the tissues which have a far superior strategy when compared with computed tomography. So this makes this strategy an exceptionally extraordinary one for the brain tumor extraction. In this paper an improvement to the watershed changes for precisely recognizing the area of brain tumor in MR pictures. This research paper is composed as takes after. In Section III, the proposed strategy is described. Experimental results and examination with existing extraction calculations are exhibited in Section IV. At last, conclusions and discussions come in Section V.

\section{RELATED WORK}

J.Z.Shah, S.A.Husain has proposed a fuzzy based versatile BPNN learning algorithm of brain MR Images. The fuzzy based versatile control procedure has been utilized for the first time as algorithm part of the different segmentation issues that has demonstrated huge impact on the learning proficiency of the BPNN. For the elimination of the additional cranial parts of the cerebrum, another and solid morphological strategy has been utilized. The results of the segmentation have been compared with the radiologist marked ground truth.

Wei Zhao and Mei xie has developed a customized skull stripping strategy in light of morphological processing. In this paper, skull-stripping in view of edge detection is examined in point of interest. The strategy can take out the brain tissue from an ordinary MRI rapidly and precisely. Finally, a request of morphological and associated part administrator to ensure the area is closed.

Jin liu, Jianxin wang has proposed a Survey of MRI based brain tumor segmentation technique where segmentation aim to separate the distinctive tumor tissues, for example, dynamic cells, necrotic center, and edema from ordinary brain tissues of White Matter (WM), Gray Matter (GM), and Cerebrospinal Fluid (CSF). The reason of this paper is to give a far reaching outline to MRI-based mind tumor segmentation techniques. Besides, the appraisal and support of the consequences of MRI-based brain tumor segmentation are discussed about.

R.B.Dubey and M.Hanmandlu has urbanized an assessment of three strategies for MRI Brain tumor segmentation. for the most part, segmentation is performed physically in clinical environment that is administrator subordinate and exceptionally dull and tedious labour intensive work. An examination of three distinctive semicomputerized strategies, viz., altered slope greatness area developing technique (MGRRGT), level set and a marker controlled watershed strategy is attempted here for 
assessing their relative execution in the segmentation of tumor.

Hui tang, Huangxiang Liu has created Tumor partition from single MRI pictures of human brain. Discovery of brain tumors might help in determination, treatment arranging. The future strategy for the brain tumor segmentation comprises of three stages. In the initial step, we standardize the picture power and enroll to a standard brain space. Second, we accomplish a pixel-wise order utilizing an irregular backwoods characterization technique. Taking after the pixel-wise grouping, we assist avoid false encouraging points morphologically. The last division is assessed utilizing Dice Similarity Coefficient, which is $78.8 \%$ for second rate tumors and $83.0 \%$ for high review tumors.

\section{THE PROPOSED SYSTEM}

Segmentation is the first and foremost step in image analysis. Specifically, the segmentation problem is defined as sufficiently partitioning an image into non-overlapping regions. The level to which this subdivision is carried is influenced by the type of the problem being solved. The segmentation end when the object of interest in the application has been isolated. The segmentation renders the image in such a form where it is easy and meaningful to analyse an image. Image segmentation is very specifically used for identifying objects and boundaries in an image. Image segmentation algorithms assign a label to every pixel in an image region, such that all the pixels with the same label share certain similar visual characteristics. The segmentation process results in a set of segments that together combine into the entire image, or a set of contours or edges extracted from the image. Each of the pixels in a region has some similarity with respect to some characteristic feature or computed attribute, such as colour, intensity or texture while adjacent regions have significantly different characteristics. The goal of segmentation is to simplify the representation of an image in to something that is more meaningful and easier to analyze. Image segmentation is done in two ways, edge based and region based. Edge based segmentation works by detecting discontinuities in the image and uses those discontinuities as the outline of each segment. Region based segmentation works by grouping similar valued adjacent pixel in an image.

Segmentation is done utilizing following strategies.

1) Threshold Segmentation: Threshold segmentation is one of the most straightforward division techniques. The input gray scale image is changed over into a binary format. The strategy depends on a limit quality which will change over gray scale image into a binary image format. The primary logic is the choice of an threshold value.

2) Watershed Segmentation: It is one of the best strategies to gathering pixels of a picture on the premise of their intensities. Pixels falling under comparative intensities are assembled together. It is a decent segmentation method for partitioning a picture to independent a tumor from the image. Watershed transform [2] is a most efficient segmentation system originating from the field of numerical morphology. The intuitive thought of this change is very basic: in the event that we consider the image as a scene or topographic alleviation, where the stature of every point is straightforwardly identified with its dim level, and consider rain progressively falling on the landscape, then the watersheds are the lines that different the "lakes" really called catchment bowls that shape. The watershed change is figured on the gradient of the first picture, so that the catchment bowl limits are situated at high slope focuses. This change has been broadly utilized as a part of numerous fields of picture preparing, including medicinal MR picture division, because of the quantity of points of interest that it has: it is very basic, instinctive, quick, parallelized system and produces a complete segmentation of the picture in isolated locales regardless of the fact that the differentiation is poor, hence staying away from the requirement for any sort of shape joining. Some essential disadvantages associated to the watershed change are the over division and poor discovery of critical ranges with low difference limits that ordinarily brings about MR brain images.

Watershed is a scientific morphological working apparatus. Watershed is ordinarily utilized for checking yield as opposed to utilizing as a data segmentation technique since it normally experiences over segmentation and under segmentation.[14]

3) Morphological Operators: After changing over the image in the binary arrangement, some morphological operations are connected on the changed over image. The reason for the morphological administrators is to isolate the tumor part of the image. Presently just the tumor part of the picture appeared as white shading. This portion has the most elevated force than different areas of the picture. Morphological administrators are connected after the watershed segmentation. Mathematical Morphology (MM) is a nonlinear sub-branch of the sign handling field which manages the use of set hypothesis ideas to the space of image examination. Morphological administrators are nonlinear changes, which adjust geometric properties of images. The operators utilize an organizing component of specific shape and size to change the image through a progression of cycles.

Morphological operations are characterized by set number juggling. In this manner, the picture which is to be handled by morphology hypothesis must be initially changed into set. Scientific morphology operations utilizes organizing component, which is normal for certain structure and highlight, to gauge the state of picture and after that complete picture preparing. In view of set hypothesis, numerical morphology is the operation which changes starting with one set then onto the next. The point of this change is to seek the extraordinary set structure of unique set. The changed set incorporates the data of the uncommon set structure and the change is acknowledged by exceptional organizing component. Consequently, the 
outcome is correlative to a few attributes of organizing component. Among all morphological operators, the opening and closing operator are crucial. They can be utilized for image depiction and in addition picture control (with commotion separating as an exceptional case). Given their sensibility to noise, in recent literature, a few recommendations were distributed to enhance their robustness. The majority aim to improve their performance as a filter.

Morphological edge detection algorithms usually select an appropriate structuring element to process an image and by making use of the basic morphological theory that include operations such as erosion, dilation, opening and closing, and synthesization operations to get a clear edge image. In detail, the synthesized mode of the operations reflects the relation between the processed image and origin image, and the selection of structuring element decides the effect and precision and the result. Therefore, the keys of morphological operations can be generalized for the design of morphological filter structure and the selection of structuring element. In medical image edge detection, it is needed to select appropriate structuring element by texture features of the image. And the size, shape and direction of structuring element must be considered roundly. Usually, except for special demand, select structuring element by $3 \times 3$ square.

By the operation elements of morphology, erosion and enlargement operations fulfil:

$\mathrm{F} \Theta \mathrm{B} \subseteq \mathrm{F} \subseteq \mathrm{F} \oplus \mathrm{B}$

Opening and closing operations fulfil:

$\mathrm{F}$ o $\mathrm{B} \subseteq \mathrm{F} \subseteq \mathrm{F} \bullet \mathrm{B}$

The standard filters which are used to eliminate the noise and the results after using these filter was compared with the proposed methodology in this work.
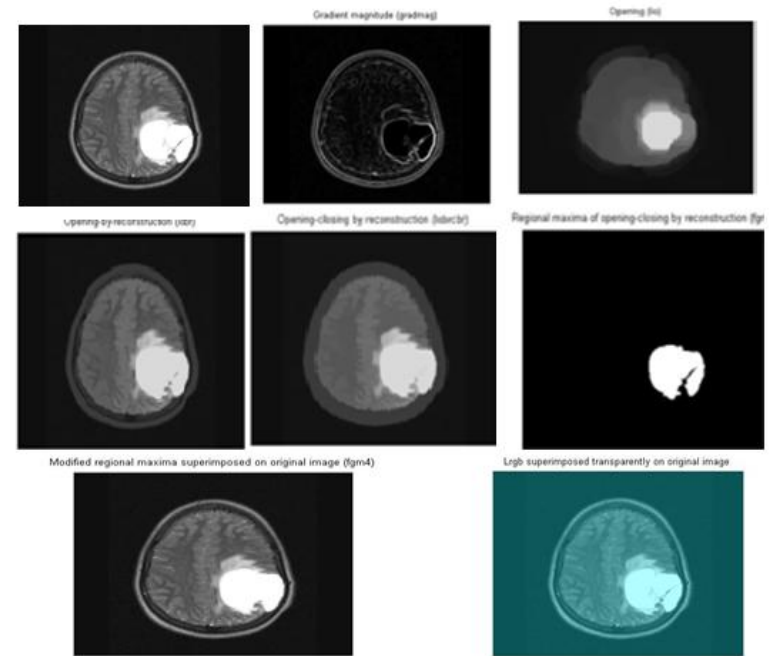

Fig.1. (a) Original image (b) Gradient magnitude (c) Opening image (d) opening by reconstruction (e) opening closing by reconstruction (f) regional maxima of opening closing image (g)modified regional maxima superimposed on original image (h) Lrgb superimposed on original image.
The filters such as averaging, Gaussian, Laplacian, Sobel, Prewitt, Log and unsharp are used. The above methodology is connected to DICOM tumor pictures without noise and same steps are executed to images subsequent to including four noises independently and resultant images are obtained. The Performance of various operators is assessed in view of the parameters viz., Correlation Coefficient, Peak Signal-to-Noise proportion and Mean square error. These parameters are figured between the separated ROI from original image and extricated ROI tumor region from noise included original image and the results of the proposed method are shown in fig 1.

IMAGE NOISE AND EVALUATION PARAMETERS In general noise is considered as undesired information which adds to the desired components and deteriorates the quality of the image. In image processing, noise $\eta(x, y)$ is defined as a process which affects the quality of the acquired image $f(x, y)$ and is not part of the scene (initial signal $-\mathrm{s}(\mathrm{x}, \mathrm{y}))$. Based on the additive noise degradation model, this process of noise addition can be written as:

$$
f(x, y)=s(x, y)+\eta(x, y)
$$

There are various image noise sources. In the process of acquiring digital images the sensors will convert optical signals into electrical signals and later into digital signals. The noise may be added in digital images during this process stated earlier. Unpredictable changes in the intensities of the pixels may be introduced at various steps involved in the acquisition process. These changing intensity values are characterised as noise. The ability in resisting the effect of the noise by various filters can be tested by corrupting an image with various noises. Depending on the nature of the noise it is categorised as image independent noise and Image dependent noise.

\section{A. Image Independent Noise}

Independent noise is described completely by an additivenoise model, where the image acquired $f(x, y)$ is the sum of original image $\mathrm{o}(\mathrm{x}, \mathrm{y})$ and the noise component $\eta(\mathrm{x}, \mathrm{y})$. Zero mean and variance is used to describe this type of noise and is referred as Gaussian noise. Signal-to-Noise ratio parameter is used to describe the impact of noise component on the original image component. In additivenoise case, the noise is considered to be uniformly distributed over the frequency domain and is predominant at higher frequencies.

\section{B. Image Dependent Noise}

i. Poisson Noise

Image detectors and recorders have a nonlinear response resulting in a noise called Poisson noise. The image data dependency on noise arises because the acquisition process that include detection and recording processes involve random emission of electron have a Poisson distribution function with mean response value. Since both the mean and variance parameters of a Poisson distribution are equal, and if noise is assumed to have unity variance, 
the signal dependent term is described by standard deviation

\section{ii. Detector Noise}

The detector noise is the type of Gaussian noise that occurs in all recorded images to some extent. The reason for this noise is that each imaging system records an image by counting photons i.e., radiation is discrete in nature. With assumption that radiation is discrete in nature, this noise can be modelled with an independent, additive model, where the noise component has zero-mean Gaussian distribution function described by its variance or standard deviation.

\section{iii. Speckle Noise}

Another important type of noise resulting in data dropouts during transmission of image data is speckle noise is caused by transmission errors. The pixels corrupted by speckle noise are set to the maximum intensity in the image, and it appears like a snow pattern in image or have single bit flip over.

\section{iv. Salt and Pepper Noise}

This noise is special case of data dropout noise and caused by errors occurring during image data transmission. This noise is characterised by adding every single pixels in the image alternatively to minimum or to the maximum intensity value in the image considered, changing the appearance of the image as salt and pepper. The percentage of pixels from the total population of the image pixels is used to quantify the effect of noise corrupted to this noise.

\section{Evaluation Parameters \\ i. Cross Correlation}

A standard means of estimating the degree of similarity between two different images or series or signals is defined as cross correlation that can be computed using equation (2) below.

$$
r=\frac{\sum_{i}\left[\left(x(i)-m_{x} *\left(y(i-d)-m_{y}\right)\right]\right.}{\sqrt{\sum_{i}\left(x(i)-m_{x}\right)^{2}} \sqrt{\sum_{i}\left(y(i-d)-m_{y}\right)^{2}}}
$$

\section{ii. PSNR (Peak Signal-to-Noise Ratio)}

Objective fidelity criteria is a subjective measure for assessing the quality of recovered image from noise corrupted image. Most commonly used parameter to measure or estimate the closeness between two data sets is the Root-Mean-Squared-Error, or RMSE . When ground truth information about the data sets considered is available, the two data sets could be the denoised to obtain an approximate or estimate image $\mathrm{f}^{\prime}(\mathrm{x}, \mathrm{y})$ of the original image $f(x, y)$. The RMSE value can be calculated by.

$$
\text { RMSE }=\sqrt{\frac{1}{X Y} \sum_{y=0}^{Y-1} \sum_{x=0}^{X-1}\left[f^{\prime}(x, y)-f(x, y)\right]^{2}}
$$

The RMSE value is proportional to the difference between two images $f^{\prime}(x, y)$ and $f(x, y)$. In case if the two images (approximate and original) are exactly same then the value is zero. In case if zero-mean additive Gaussian noise is considered, the RMSE value between the noisy image and original image will be exactly equal to the standard deviation of noise. Peak Signal-to-Noise ratio, or PSNR is related to and derived from RMSE, and measured in decibels $(\mathrm{dB})$. This logarithmic measure of PSNR in terms of RMSE is computed using equation (4) where z-1 is the maximum possible pixel intensity.

$$
P S N R=20 \log _{10}\left(\frac{z-1}{R M S E}\right) d B
$$

\section{iii. Mean Absolute Error}

PSNR and RMSE use the square of the pixel differences of two images resulting in large errors frequently. An alternate measure to improve the potential problem of errors occurrence can be achieved by taking the average of the absolute error, or MAE and calculated using equation (5) . By computing MAE through mean of absolute error, the magnitude errors are overcome, and hence it is less likely biased by large occasional errors compared to RMSE.

$$
M A E=\frac{1}{X Y} \sum_{y=0}^{Y-1} \sum_{x=0}^{X-1}\left|f^{\prime}(x, y)-f(x, y)\right|
$$

\section{IV.EXPERIMENTAL RESULT ANALYSIS AND DISCUSSIONS}

Two sorts of experiments are done to assess the execution of the proposed approach qualitatively and quantitatively. The performance measures used to analyze the execution are PRI (Probabilistic Rand Index), VOI (variation of information) \&GCE (global consistency error). PRI assesses the pair wise connections between pixels of divided result and numerous ground-truth divisions and takes values in the reach $(0,1)$. Hence higher PRI esteem shows a superior match between the sectioned result and the ground-truth information. VoI, GCE are error measures that have to be reduced by the good segmentation algorithm.

i. Rand Index (RI)

The Rand Index (RI) checks the part of pairs of pixels whose marking are reliable between the computed segmentation and the ground truth averaging over numerous ground truth segmentations. The Rand measure is a measure of the similitude between two data clusters. Given a set of $\mathrm{n}$ components and two partitions of $\mathrm{S}$ to look at, and, we characterize the following:

a. The quantity of pairs of elements in $S$ that are in the same set in $\mathrm{X}$ and in the same set in $\mathrm{Y}$

b. The quantity of pairs of elements in $S$ that are in different sets in $\mathrm{X}$ and in distinctive sets in $\mathrm{Y}$

c. The quantity of pairs of elements in $S$ that are in the same set in $\mathrm{X}$ and in diverse sets in $\mathrm{Y}$ 
Vol. 5, Issue 12, December 2016

d. The quantity of sets of elements in $S$ that are in different sets in $\mathrm{X}$ and in the same set in $\mathrm{Y}$

The Rand Index (RI) defined as

$$
R=(a+b) /(a+b+c+d)=(a+b) /((n / 2))
$$

Where $\mathrm{a}+\mathrm{b}$ as the number of agreements between $\mathrm{X}$ and $\mathrm{Y}$ and $\mathrm{c}+\mathrm{d}$ as the number of disagreements between $\mathrm{X}$ and $\mathrm{Y}$. The Rand index has a value between 0 and 1 , with 0 indicating that the two data clusters do not agree on any pair of points and 1 indicating that the data clusters are exactly the same.

\section{ii. Variation of Information (VOI)}

The Variation of Information (VOI) metric characterizes the distance between two segmentations as the average conditional entropy of one segmentation given the other, and in this manner measures the measure of randomness in one segmentation which can't be clarified by the other. Assume we have two clustering (a division of a set into a several subsets) $\mathrm{X}$ and $\mathrm{Y}$ where

$$
\mathrm{X}=\{\mathrm{X} 1, \mathrm{X} 2 \ldots \mathrm{Xk}\}, \mathrm{pi}=|\mathrm{Xi}| / \mathrm{n}, \mathrm{n}=\Sigma \mathrm{k}|\mathrm{Xi}| \text {. }
$$

At that point the variety of information between two clustering is:

$$
\mathrm{VI}(\mathrm{X} ; \mathrm{Y})=\mathrm{H}(\mathrm{X})+\mathrm{H}(\mathrm{Y})-2 \mathrm{I}(\mathrm{X}, \mathrm{Y})
$$

Where, $\mathrm{H}(\mathrm{X})$ is entropy of $\mathrm{X}$ and

$\mathrm{I}(\mathrm{X}, \mathrm{Y})$ is mutual information between $\mathrm{X}$ and $\mathrm{Y}$. The common data of two clustering is the loss of uncertainty of one grouping if the other is given. Consequently, shared positive and limited by $\{\mathrm{H}(\mathrm{X}), \mathrm{H}(\mathrm{Y})\}_{-} \log 2(\mathrm{n})$.

\section{iii. Global Consistency Error (GCE)}

The Global Consistency Error (GCE) measures the degree to which one segmentation can be seen as a refinement of the other. Segmentations which are connected are thought to be steady, since they could represent to the same image segmented at distinctive scales. Segmentation is essentially a division of the pixels of a picture into sets. The portions are sets of pixels. If one segment is appropriate subset of the other, then the pixel lies in an area of refinement, and the error should be zero. If there is no Subset relationship, then the two regions overlap in an Inconsistent manner.

$$
\tau=\sum_{j=1}^{k} \sum_{i=1}^{x}=1\left\|X^{(j)}-\Gamma_{j}^{j}\right\|^{2} \text { where }\left\|X^{(j)}-C_{j}\right\|^{i}
$$

Where, segmentation error measure takes two divisions S1 furthermore, $\mathrm{S} 2$ as input, and produces a genuine esteemed output in the reach [0::1] where zero signifies no error. For a given pixel pi consider the segments in S1 and S2 that contain that pixel.

iv. Calculation the tumor region

The tumor area region is calculated by the following equation:

Tumor area $=\mathrm{A}$ total number of pixel in the tumor region
$\mathrm{A}=\mathrm{V} \times \mathrm{H}$

Where, $A=$ the area of each pixel

$\mathrm{H}=$ horizontal dimension of the image

$\mathrm{V}=$ vertical dimension of the image

\section{v. PEAK SIGNAL TO NOISE RATIO(PSNR)}

The Peak Signal to Noise Ratio (PSNR) is used to find the deviation of segmented image from the ground truth image. Equation (8) represents the PSNR. In this equation mean squared error (MSE) for two $\mathrm{M} * \mathrm{~N}$ monochrome images $f$ and $z$ and it is given by Equation (9). MaxBits gives the maximum possible pixel value (255) of the image.

$$
\begin{aligned}
& P S N R=10 X \log _{10} \frac{\text { MaxBits }^{2}}{M S E} \\
& M S E=\frac{1}{M x N} \sum_{x=0}^{M-1} \sum_{y=0}^{N-1}\left((f(x, y)-z(x, y))^{2}\right.
\end{aligned}
$$

\section{vi. STRUCTURAL SIMILARITY}

Structural Similarity Index (SSIM) is a method for measuring the similarity between two images. The SSIM is measured between two windows $\mathrm{X}$ and $\mathrm{Y}$ of common size $\mathrm{N}^{*} \mathrm{~N}$ on image using Eq. (3).

$$
\operatorname{SSIM}(x, y)=\frac{\left(2 \mu_{x} \mu_{y}+c_{1}\right)\left(2 \sigma_{x y}+c_{2}\right)}{\left(\mu_{x}^{2}+\mu_{y}^{2}+c_{1}\right)\left(\sigma_{x}^{2}+\sigma_{y}^{2}+c_{2}\right)}
$$

The amount of quantitative information available on medical images is enormous. Computer quantification may hold more potential than computerised detection. The main reason that most radiolilogic scoring systems are not used routinely in clinical practice that they are too time consuming and cumbersome to apply. For effective and widespread use of any cad system it should be integrated in to through workflow of radiologist and interaction should be intuitive and the results should available instantaneously.

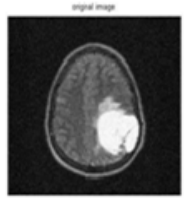

(a)

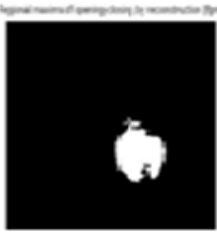

(e)

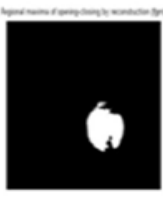

(b)

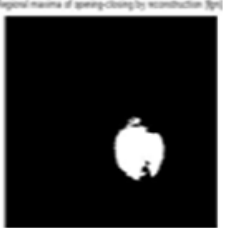

(f)

(c)
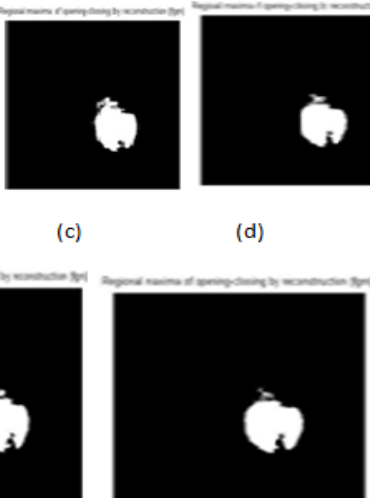

(g)
Fig. 2. (a) DICOM image with Gaussian noise b) ROI using average filter c) ROi using Gaussian filter d) ROI using Laplacian filter e) ROI using sobel filter f) ROI using Log filter g) ROI using unsharp filter. 
Vol. 5, Issue 12, December 2016

Current systems for computer aided detection have been intruded as complementary tools that draw the radiologist attention to images that need further evaluation. The proposed calculations have been implemented utilizing MATLAB. The execution of different image segmentation methodologies are analyzed and talked about. The measurement of image segmentation is hard to measure. There is no regular algorithm for the image segmentation. The measurable estimations could be utilized to measure the nature of the image segmentation. The rand index (RI), global consistency error (GCE) \& VOI are utilized to assess the execution. The detailed description with formulae of RI, GCE, VOI parameters are clarified in point of interest as follows.

After obtaining the results the evaluation process involves comparing the original region segmented and tumor detected with the MRI images for various noise and various methods. The parameters used are correlation coefficient, peak signal to nose ration. The values are tabulate in the Table 1, 2 and 3.

TABLE.1. Correlation Coefficient values between RoI segmented image with and without noise.

\begin{tabular}{|c|c|c|c|c|c|c|c|}
\hline Noise & Sobel & Prewitt & Average & LoG & Gaussian & Laplacian & Unsharp \\
\hline Salt and Pepper & 0.9963 & 0.9924 & 0.9942 & 0.9949 & 0.9942 & 0.9960 & 0.9949 \\
\hline Speckle & 0.8604 & 0.7684 & 0.7764 & 0.8536 & 0.7656 & 0.7769 & 0.7755 \\
\hline Gaussian & 0.8167 & 0.8987 & 0.8131 & 0.8903 & 0.9013 & 0.8028 & 0.8012 \\
\hline Poisson & 0.9200 & 0.9113 & 0.9163 & 0.8972 & 0.8287 & 0.8262 & 0.8289 \\
\hline
\end{tabular}

TABLE.2. PSNR values between ROI segmented image with and without noise.

\begin{tabular}{|l|l|c|c|l|c|c|c|}
\hline Noise & Sobel & Prewitt & Average & LoG & Gaussian & Laplacian & Unsharp \\
\hline $\begin{array}{c}\text { Salt and } \\
\text { Pepper }\end{array}$ & 92.31 & 89.094 & 90.275 & 90.854 & 90.275 & 91.902 & 90.854 \\
\hline Speckle & 76.57 & 74.644 & 74.788 & 76.383 & 74.607 & 74.081 & 73.997 \\
\hline Gaussian & 75.55 & 77.860 & 74.71 & 77.45 & 77.92 & 74.34 & 75.20 \\
\hline Poisson & 78.89 & 78.44 & 78.77 & 77.52 & 75.16 & 74.97 & 75.16 \\
\hline
\end{tabular}

TABLE.3. MAE values between ROI segmented image with and without noise.

\begin{tabular}{|c|c|c|c|c|c|c|c|}
\hline Noise & Sobel & Prewitt & Average & LoG & Gaussian & Laplacian & Unsharp \\
\hline $\begin{array}{c}\text { Salt and } \\
\text { Pepper }\end{array}$ & 0.000 & 0.0001 & 0.0001 & 0.0001 & 0.0001 & 0.0000 & 0.0001 \\
\hline Speckle & 0.0014 & 0.0022 & 0.0022 & 0.0015 & 0.0023 & 0.0025 & 0.0026 \\
\hline Gaussian & 0.0018 & 0.0011 & 0.0022 & 0.0012 & 0.0010 & 0.0024 & 0.0020 \\
\hline Poisson & 0.0008 & 0.0009 & 0.0009 & 0.0011 & 0.0020 & 0.0021 & 0.0020 \\
\hline
\end{tabular}

The above mentioned aspects can be taken as future work to design a complete CAD for early detection of brain tumors in situations where the images are added with image noise.

The Table 4 performance analysis revieals that the PRI, SSIM values are higher and VOI and GCE values are lower for morphology based segmentation algorithm compared to other above mentioned algorithms.
TABLE.4. Performance evaluation

\begin{tabular}{|c|l|c|c|c|c|}
\hline & \multicolumn{1}{|c|}{ METHODS } & PRI & VOI & GCE & SSIM \\
\cline { 2 - 6 } & Watershed method & 0.58 & 1.02 & 0.07 & 0.338 \\
\cline { 2 - 6 } IMAGE & K Means & 0.97 & 0.12 & 0.0161 & 0.958 \\
\cline { 2 - 6 } & Proposed method & 0.987 & 0.116 & 0.0164 & 0.96 \\
\hline \multirow{4}{*}{ IMAGE 2 } & Watershed method & 0.606 & 1.335 & 0.197 & 0.144 \\
\cline { 2 - 6 } & K means & 0.983 & 0.15 & 0.011 & 0.881 \\
\cline { 2 - 6 } & Proposed method & 0.962 & 0.540 & 0.013 & 0.90 \\
\hline \multirow{4}{*}{ IMAGE 3 } & Watershed method & 0.5058 & 1.272 & 0.029 & 0.277 \\
\cline { 2 - 6 } & K means & 0.991 & 0.064 & 0.0052 & 0.968 \\
\cline { 2 - 6 } & Proposed method & 0.975 & 0.0867 & 0.004 & 0.967 \\
\hline \multirow{4}{*}{ IMAGE 5 } & Watershed method & 0.515 & 1.29 & 0.034 & 0.29 \\
\cline { 2 - 6 } & K means & 0.984 & 0.094 & 0.009 & 0.956 \\
\cline { 2 - 6 } & Proposed method & 0.970 & 0.0867 & 0.018 & 0.965 \\
\hline & Watershed method & 0.580 & 1.21 & 0.03 & 0.30 \\
\cline { 2 - 6 } & K means & 0.887 & 0.06 & 0.012 & 0.891 \\
\cline { 2 - 6 } & Proposed method & 0.984 & 0.1887 & 0.019 & 0.920 \\
\hline
\end{tabular}

TABLE.5. Average Calculation of Performance Analysis method

\begin{tabular}{|l|l|l|l|l|}
\hline METHODS & PRI & VOI & GCE & SSIM \\
\hline Watershed method & 0.5573 & 1.225 & 0.072 & 0.269 \\
\hline K-Means & 0.9608 & 0.196 & 0.010 & 0.929 \\
\hline Proposed method & 0.9752 & 0.191 & 0.01 & 0.94 \\
\hline
\end{tabular}

The average of results is shown in Table 5. If the value of PRI is higher and GCE, VOI are lower than the segmentation approach is better.

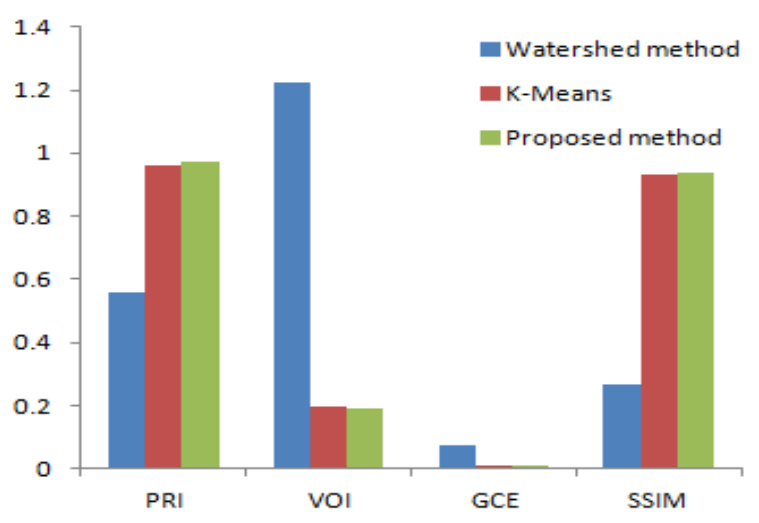

Fig. 3. Performance analysis chart

The Fig. 3 average performance analysis chart reveals that the rand index of proposed method is higher than others and also the global consistency error, variation of information, are lower than others.

\section{CONCLUSION}

Detection of tumor is one of the significant issues for diagnosis of the cancer. In the event that it is identified in right on time stages builds the survival rate. This paper presented an improvement to the segmentation for the 
Vol. 5, Issue 12, December 2016

extraction of brain tumor of MR images based on morphological operators and watershed segmentation. The validity of the work is performed by applying the procedure on MRI with various noises. This makes simple approach to identify and study the brain tumor. This strategy can be used as an initial Step for computer aided diagnosis to assess the execution of different edge detection techniques. The assessment of various edge identifying strategies is performed on MRI brain image for recognizing the ROI with four sorts of noises. From visual examination of the outcomes and considering the parameters the correlation coefficient, peak signal to noise ratio and mean absolute error it can be seen that the Gaussian operator performs better in the Gaussian noise environment. For salt and pepper noise, poission noise and speckle noise additions sobel operator exhibits better performance. The performance of segmentation algorithm is measured using segmentation parameters PRI, GCE, VOI, and PSNR, SSIM and shown the best results for Proposed method. The operators that were evaluated include Average, Gaussian, Laplacian, Sobel, Prewitt, LoG and Unsharp. These operators were evaluated for performance under different types of noise like Salt and Pepper, Gaussian, Poisson and Speckle noise. From the results, it can be observed that the Sobel operator provides a better performance of increased PSNR, reduce MAE and high correlation for all types of noises excluding the Gaussian noise. It can also be observed that the proposed method delivers expected performance terms of extracting the Region of Interest (ROI) even in the presence of different types of noises. This feature is very essential to make the proposed tool robust in the presence of noise.

\section{REFERENCES}

[1] V. Grau, A.U.J.Mewes, M.Alcañiz, "Improved Watershed Transform for Medical Image Segmentation Using Prior Information," IEEE Transactions On Medical Imaging, Vol. 23, No. 4, pp 447-458, June 2004.

[2] A. Mustaqeem, A.Javed, T. Fatima, "An Efficient Brain Tumor Detection Algorithm Using Watershed \& Thresholding Based Segmentation," International Journal of Image, Graphics and Signal Processing,Vol.10, No.3, pp34-39, 2012.

[3] T.Logeswari,M.Karnan,et.al,"Animproved Implementation of brain tumor detection using segmentation based on soft computing," Journal of Cancer Research and Experimental Oncology, Vol. 2,No.1,pp 06-4, 2010.

[4] M. Brummer, R. Mersereau, R. Eisner, and R. Lewine, "Automatic detection of brain contours in MRI data sets," IEEE Trans. Med.Imag.,Vol. 1No.4, pp153-166,1993.

[5] M.S. Atkins and B.T. Mickiewicz, "Fully Automatic Segmentation of the Brain in MRI," IEEE Tran. On medical imaging, Vol. 17,No.1, pp 98-107,1998.

[6] Devos, A, Lukas, L., Does, "The combination of magnetic resonance imaging and spectroscopic imaging improve the classification of brain tumor Engineering in Medicine and Biology Society," Annual International Conference of the IEEE, vol.1,No.5 Sept. 2004.

[7] Farmer, M.E, Jain, A.K., "A wrapper-based approach to image segmentation and classification, Image Processing," IEEE Transactions on journals and magazines, pp2060 - 2072,Dec 2005.

[8] Senthilkumaran N, Kirubakaran C, A Case Study on Mathematical Morphology Segmentation for MRI Brain Image, International Journal of Computer Science and Information Technologies, Vol. 5,No.4, pp 5336-5340,2014.
[9] Hall LO, Bensaid AM, Clarke LP,Velthuizen RP, Silbiger MS, Bezdek, " A comparison of neural network and fuzzy clustering techniques in segmenting magnetic resonance images of the brain," IEEE Trans. Neural Networks,Vol. 3No.5,pp 672-681,1992.

[10] Gang Li , "Improved watershed segmentation with optimal scale based on ordered dither halftone and mutual information," Computer Science and Information Technology (ICCSIT), 3rd IEEE international Conference, 9-11 July 2010.

[11] J.Mehena, "Medical Image edge detection based on mathematical morphology," International Journal of Computer and communication technology, Vol.-2,No.6, pp 45-48,2011.

[12] M.C. Christ, R. Parvathi, "Segmentation of Medical Image Using Clustering and Watershed Algorithms," American Journal, Vol.8, No.12, 2011, pp1349-1352.

[13] Sathya B, Manavalan R, "Image Segmentation by Clustering Methods Performance Analysis," International Journal of Computer Appl. Vol.29, No.11,pp27-32,2013.

14] Raja SVK, Abdul Khadir AS, Ahamed S SR. "Moving toward Region-Based Image Segmentation Techniques," J Theor Applied Information Technology, Vol. 5,No.9,pp81-7,2009.

[15] Kananga T, Mount, DM, Piatko CD, Silverman, R, Wu, AY, “An Efficient k-Means Clustering Algorithm: Analysis and Implementation," IEEE Trans Pattern Anal Mach Intel. Vol.24, No.7, pp.881-92.

[16] Wei Zhao, Mei Xie, Jingjing Gao, and Tao Li, “A modified skullstripping method based on Morphological Processing", Second International Conference on Computer Modeling and Simulation, pp. $159-163,2014$

[17] Jin Liu, Min Li, Jianxin Wang, Fangxiang Wu, Tianming Liu, and Yi Pan, "A Survey of MRI-Based Brain Tumor Segmentation Methods," tsinghua science and technology,pp. 578-595, 2014.

[18] Gopi Karnam and Ramashri Thirumala "design and development of a computer aided diagnosis system for segmentation of brain tumor " in ARPN Journal of Engineering and Applied Sciences Vol. 10, NO 22, December, 2015, ISSN 1819-6608

\section{BIOGRAPHIES}

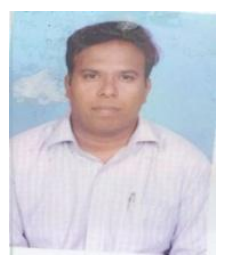

K. Gopi (Karnam Gopi) obtained his Bachelor's degree in Electronics and Communication Engineering form periyar University. Then he obtained his Masters degree in Applied Electronics and currently Pursuing $\mathrm{PhD}$ in Electronics and communication majoring in Digital Image Processing in Sri Venkateswara University, Tirupati. He is Working as an Asst.professor in Department of ECE, Sreenivasa Institute of Technology and management studies, chittoor.He is having 12 years of teaching experience\& his area of interest include Digital image processing(image segmentation).

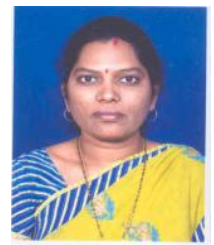

Dr. T. Ramashri (Tirumala.Ramashri) received the B.Tech degree from the Karnataka University and the M.Tech degree from the Sri Venkateswara University, Tiurpati. and the Ph.D. degree from the Sri Venkateswara University, Tiurpati she is working as Professor in ECE Department S.V University College of engineering, Tirupati.She is having more than 18 years Teaching Experience. She published more than 14 papers in various national and international journals and conferences, and has delivered many special lectures and interested in Digital Image Processing. She is a Life member of ISTE, Life member of IETE. 